\title{
Metric System
}

National Cancer Institute

\section{Source}

National Cancer Institute. Metric System. NCI Thesaurus. Code C42540.

An international decimal system of weights and measures, in which the basic units are the meter for length, the gram for weight, and the liter for volume. The metric system units have to satisfy three conditions: one fundamental unit is defined for each quantity; multiples and fractions of fundamental units are created by adding prefixes (denoting powers of ten) to the names of the defined units; the fundamental units are defined rationally and are related to each other in a rational fashion. The metric system evolved into the International System of Units (Systeme International d'Unites, SI). 